\title{
Varia \\ La prévention des préjugés flagrants et voilés perçue par les élèves : lorsque les enseignant-e.s ont leur mot à dire
}

\author{
Alessandro Bergamaschi, Catherine Blaya, Jimmy Stef, Université Côte d'Azur \\ Francesco Arcidiacono, Haute Ecole Pédagogique - BEJUNE
}

Cet article sintéresse aux préjugés "flagrants " et "voilés " manifestés par 1'858 élèves de collège et de lycée en France au moyen d'une enquête par questionnaire. Une première analyse descriptive permet de repérer les facteurs sociaux et scolaires pouvant influencer la perception par les élèves de l'investissement des enseignant.e.s autour de questions liées à l'immigration. À partir de modèles multivariés, les analyses montrent que lorsque les élèves déclarent que leurs enseignant.e.s abordent les thématiques liées au racisme, aux discriminations et à la diversité culturelle, les attitudes intolérantes baissent. L'analyse causale met en exergue l'existence de facteurs externes facilitant l'appropriation des interventions des enseignant.e.s par les élèves.

\section{Objet et problématique}

Les préjugés, les manifestations d'intolérance et la xénophobie envers les minorités dites immigrées représentent une question ouverte au sein de l'espace européen. En effet, la diffusion de l'intolérance envers les populations immigrées auprès des jeunes générations est actuellement l'un des aspects les plus problématiques de nos sociétés (European Network Against Racism [ENAR], 2015-2016).

Sanchez-Mazas et Fernandez-Iglesias (2002) soulignent l'importance d'aborder les questions liées à la différence culturelle en milieu scolaire, afin d'éduquer les élèves à une vision moins stéréotypée de l'Autre. Ainsi, enquêter sur le rapport des adolescent.e.s à ces enjeux et s'intéresser au rôle potentiel des enseignant.e.s dans la perception de ces phénomènes représentent un intérêt de premier ordre. À la suite des derniers attentats terroristes en Europe, l'importance d'une éducation à la citoyenneté autour de questions relatives à l'immigration est émergée en tant que thématique pédagogique de premier ordre (Ostinelli, 2020). Il nous semble propice, avec le recul de quelques années, de nous intéresser aux effets des interventions des enseignant.e.s sur les questions liées au racisme, aux discriminations et à la diversité culturelle sur les attitudes des jeunes. Cela représente une perspective de recherche peu explorée à ce jour.

La question de la diversité culturelle au sein de la société française, dont la philosophie politique est axée sur le principe d'égalitarisme universel, est source de débats et interroge le fonctionnement de nombre d'institutions, y compris du système éducatif. Bien que des initiatives de valorisation de la diversité culturelle voient timidement le jour, notamment de la part d'enseignant.e.s qui croient fortement dans la valeur de promouvoir toutes les identités, celles-ci n'ont jamais fait l'objet d'une validation par les institutions centrales.

Au vu de la place spécifique occupée par l'école dans la perpétuation de l'esprit citoyen, il nous semble pertinent d'interroger le rôle qu'elle peut jouer en termes de déconstruction des préjugés et des idées stéréotypées envers les minorités immigrées. Dans cette étude nous nous sommes intéressé.e-s à la perception par les élèves de la tendance de leurs enseignant.e.s à aborder en classe les questions de racisme, de discrimination et de diversité culturelle. Nous avons étudié les effets de cette perception sur leurs préjugés à caractère « flagrant » et " voilé " (Pettigrew et Meertens, 1995, 1997). Les premiers continuent à affleurer au sein des discours publics et représentent des formes d'hostilité qui ont émergé alors que le statut social et économique subalterne des minorités immigrées ne faisait pas encore l'objet de contestations de la part des sociétés d'accueil. Les préjugés voilés représentent au contraire des formes d'hostilité subtiles et moins franches qui se sont développées à partir des années 70 aux États-Unis (Gaertner et Dovidio, 1984) et dans les années 80 en Europe (Barker, 1984). Depuis, montrer du doigt les minorités immigrées en tant que menace pour des questions matérielles est devenu socialement moins acceptable. En revanche, des réflexions plus fines quant aux possibilités de cohabitation avec des identités culturelles hétérogènes et différentes par rapport aux cultures nationales des sociétés d'accueil ont commencé à gagner du terrain dans tous les milieux sociaux (De Rudder et al., 2000; Pérez, 2013). 


\section{Le lien complexe entre éducation scolaire et attitudes intergroupes}

La relation entre éducation scolaire, attitudes et représentations de la diversité est multiple. D'une part, selon la perspective des nouvelles théories structuralistes du racisme, certain.e.s auteur.e.s soutiennent que l'éducation ne ferait que transmettre des idées conformes aux logiques de domination raciste de la société (BonillaSilva, 1997). Pour les auteur.e.s qui s'inscrivent dans ce paradigme, principalement issu.e.s du contexte nordaméricain, les catégories raciales (par ex. blanc, black, hispano, etc.) sont des produits socio-historiques qui représentent le principe organisateur de la société, ses hiérarchies et ses relations sociales. Brown et Mutegi (2010) se sont principalement intéressés à la sous-représentation des noir.e.s dans le milieu de la recherche en sciences dures aux États-Unis. D'après eux, le système éducatif, en diffusant des savoirs parés de raison et de légitimité scientifique, viserait à véhiculer les principes de l'« idéologie scientifique raciste ». Les contenus des programmes d'enseignement contribueraient à diffuser l'idée de supériorité de certains groupes raciaux par rapport à d'autres, notamment des blanc.he.s sur les noir.e.s, et ainsi l'école ne transmettrait pas une représentation positive de la diversité.

La recherche en sociologie de l'éducation documente les effets de l'éducation sur les préjugés à caractère raciste. Selon cette orientation institutionnaliste, le système d'enseignement devrait véhiculer les valeurs démocratiques utiles à l'accompagnement des jeunes vers une citoyenneté réflexive (Hyman et Wright, 1979 ; Vogt, 1997). Cette approche a suscité nombre de projets sur l'éducation interculturelle dont l'objectif est de proposer aux élèves des moments de réflexion sur la citoyenneté, l'égalité, l'équité et le lien social (Ouellet, 2002 ; Pagé, 2001). D'autres recherches évoquent la "théorie de la socialisation ", à savoir l'existence d'une corrélation positive entre les valeurs et principes cumulés dans le bagage scolaire de l'individu et le niveau de tolérance envers la diversité ethno-culturelle (Coenders et Scheepers, 2003 ; Scheepers et al., 2002). D’après cette théorie, la relation inverse entre préjugés racistes et éducation serait liée à la diffusion de valeurs démocratiques par les systèmes d'enseignement (Selznick et Steinberg, 1969). D'autres études soulignent que l'instruction permet de se forger un esprit plus autonome et une pensée moins influençable par les conditionnements extérieurs : l'éducation scolaire faciliterait donc l'apprentissage de principes et valeurs qui encouragent la tolérance (Miller et al., 1985). Il s'agit d'un courant d'études qui s'est développé notamment aux États-Unis sous les termes d'éducation ou pédagogie multiculturelle (multicultural teaching; Banks, 1996) et qui s'est manifesté plus récemment en Europe (Geerlings et al., 2017 ; Verkuyten et Thijs, 2013). En particulier, dans leur étude sur le rôle des enseignant.e.s flamand.e.s dans la prévention des préjugés chez les élèves, Vervaet et al. (2018) ont montré que les perceptions des jeunes quant à l'implication des enseignant.e.s dans des activités de sensibilisation sont bien plus corrélées à leurs attitudes interethniques par rapport à ce que les enseignant.e.s affirment faire en matière d'éducation à la diversité.

Cependant, les effets de l'éducation scolaire sur les représentations des minorités immigrées ne sont pas toujours directs : d'autres facteurs peuvent entrer en jeu et complexifier cette première relation d'influence. Hello et al. (2010) ont mené une étude aux Pays-Bas sur les effets d'interaction que l'éducation scolaire et la socialisation familiale exercent sur le niveau de distance ethnique exprimé par les adolescent.e.s. Cette variable dépendante a été mesurée en demandant le degré d'acceptation ou de refus de situations caractérisées par des niveaux différents de proximité avec des minorités immigrées (voisinage, travail, loisirs, relations affectives, etc.). Les résultats montrent que, bien que le niveau scolaire des jeunes exerce des effets significatifs (les plus instruit.e.s sont plus ouvert.e.s envers les minorités), les valeurs parentales au sujet des minorités immigrées jouent aussi un rôle central. Autrement dit, les attitudes d'ouverture ou de fermeture à la diversité des jeunes sont renforcées ou bien atténuées en fonction des représentations de leurs familles à l'égard des minorités. Une étude menée par Haegel (1999) en France a montré que le niveau d'intolérance raciste est le résultat de l'interaction entre la socialisation scolaire et celle transmise par la famille. D'après l'auteure, les effets d'une éducation scolaire longue, où les individus ont eu la possibilité de s'approprier des valeurs et des connaissances utiles à une vision moins stéréotypée des minorités immigrées, sont renforcés lorsque les enfants ont des parents qui eux aussi ont effectué des scolarités longues, alors qu'ils sont atténués lorsque les parents ont effectué des études plus courtes. Il s'agit d'effets indirects exercés par les premières formes de socialisation acquises en famille. Cependant, si dans certaines familles les discussions sur de tels sujets sont à l'ordre du jour, dans d'autres, elles sont bien plus sporadiques voire inexistantes. Ces différences sont le résultat de l'interaction de plusieurs facteurs, comme le niveau d'études des parents, leur engagement civique, les caractéristiques de leurs milieux professionnels. Il est indéniable que cette première forme de socialisation laisse une empreinte tangible au niveau du système de valeurs de référence de l'individu (Lahire, 2017). 
Dans le système éducatif, les questions du rapport à la diversité culturelle et ses épiphénomènes tels que les préjugés et les discriminations sont mentionnées dans le référentiel des compétences du personnel enseignant, ainsi que dans le programme de certaines matières telles que l'éducation morale et civique, ou encore l'éducation à la citoyenneté. Toutefois, il s'agit toujours de thématiques à forte teneur idéologique pour lesquelles il est parfois difficile de prendre des distances par rapport à sa propre vision du monde. Certain.e.s enseignant.e.s peuvent y accorder une attention élevée alors que d'autres beaucoup moins, voire tenir des propos intolérants (Perroton, 2000). En effet,

le niveau d'adhésion des enseignant.e.s vis-à-vis de l'éducation à la tolérance et à la diversité varie en fonction de leurs propres systèmes de valeurs et de leurs orientations politiques et influence donc la mise en œuvre

de valeurs multiculturelles dans leurs pratiques quotidiennes. (Bekhuis et al., 2012, p. 231, traduction libre)

Nous retrouvons un questionnement similaire dans une vaste enquête menée à échelle européenne sur la relation entre éducation scolaire et préjugés (Hagendoorn et Nekuee, 1999). Selon ces auteurs, l'éducation scolaire améliore les capacités cognitives et réduit l'ethnocentrisme des individus. Cependant, très peu de connaissances existent quant aux méthodes d'enseignement et aux pratiques mises en ouvre en classe. Dans une étude ethnographique menée en banlieue parisienne, Roussier-Fusco (2003) s'est intéressée aux stratégies de gestion des relations entre élèves déployées par des enseignant.e.s dans des classes marquées par une diversité ethnique et culturelle. Bien que la présence de conflits et d'attitudes à caractère raciste demeure constante, leur manifestation est variable en fonction de la posture choisie par les enseignant.e.s. Si le contournement des questions relatives à l'ethnicité accentue les tensions, les commenter de façon négative contribue à les banaliser alors qu'une présentation critique de l'ethnicité polarise les relations entre élèves autour de grands débats qui animent le discours public.

Aux États-Unis, un courant de recherches sur les dispositifs de prévention des préjugés racistes chez les jeunes s'est inspiré de la "théorie critique de la race ", selon laquelle le désavantage social des noir·e·s persisterait à cause d'une structure sociale où les catégories raciales sont organisées de manière hiérarchique (Bell, 1992). Par exemple, l'étude de Grosland (2019) a impliqué plusieurs classes de lycée dans un protocole prévoyant des storytellings pour renforcer le sentiment d'empathie des élèves par rapport à des situations en lien avec le racisme et les discriminations. Les résultats montrent que les jeunes qui ont assisté aux narrations faisaient preuve d'attitudes d'ouverture et d'un esprit critique plus accentués.

Ces contributions suggèrent deux réflexions majeures. Premièrement, l'éducation formelle contribue à façonner les représentations de la diversité culturelle. Cependant, la relation entre éducation scolaire et préjugés semble influencée par d'autres facteurs propres à la socialisation de l'individu au sein de la famille. Deuxièmement, la façon dont l'éducation formelle permet de réduire les attitudes d'hostilité envers les minorités immigrées demeure à ce jour une véritable "boîte noire ". La mise en ouvre du même dispositif d'éducation interculturelle peut se dérouler de manière différente selon les capacités, la formation, la motivation de chaque enseignant.e ainsi que leur positionnement idéologique face aux enjeux de l'immigration. De la même manière, il est légitime de penser que l'implication des enseignant·ess est également influencée par l'orientation générale de l'établissement scolaire envers ces mêmes questions (Varvaet et al., 2018). Autrement dit, il n'est pas aisé d'aborder en classe les enjeux que pose la diversité culturelle, car ils interrogent en effet les principes de nos sociétés démocratiques, au sein desquelles l'égalitarisme et l'universalisme sont systématiquement mis à l'épreuve par les inégalités et discriminations des minorités immigrées (Bonnery, 2006 ; Brinbaum et Primon, 2016 ; Moguérou et al., 2015 ). Ceci est particulièrement le cas dans le système éducatif français, où le corps enseignant doit, d'une part, faire preuve de neutralité axiologique alors que, d'autre part, il doit se conformer et se faire le porte-parole d'une tradition culturelle républicaine en faveur d'une « indifférence aux différences » (Duru-Bellat et van Zanten, 2012).

\section{Objectif et hypothèses}

Nous nous intéressons aux perceptions des élèves quant à la propension des enseignant·e.s à aborder en classe les questions du racisme, de la discrimination et de la diversité culturelle. Notre objectif est d'étudier les effets des perceptions sur leurs préjugés flagrants et voilés. Nous avons choisi d'interroger directement les élèves afin de contourner le risque d'obtenir des réponses normatives de la part des enseignant·e•s, en lien avec les travaux en pédagogie ou éducation multiculturelle précédemment cités. 
Dans un premier temps, nous nous focalisons sur les relations d'influence possibles entre les perceptions des élèves quant à l'investissement des enseignant.e.s et le profil social de ces jeunes. Nous nous attendons à ce que la perception des interventions des enseignant.e.s sur les questions en lien avec l'immigration soit influencée par le profil social des élèves, ainsi que par les caractéristiques des filières scolaires. Dans un second temps, nous vérifions la principale hypothèse de l'étude, à savoir les effets de ces interventions perçues sur les regards que les élèves portent sur les préjugés. Plus précisément, nous prévoyons que lorsque les élèves ont le sentiment que les enseignant.e.s abordent ces questions, cela exerce une influence positive sur la réduction de leurs préjugés flagrants et voilés. Ensuite, nous cherchons également à vérifier si les effets des interventions des enseignant.e.s perçus par les élèves peuvent être influencés par d'autres facteurs. À ce titre, et conformément à la thèse de la théorie de la socialisation, la transmission des valeurs et principes via les actions des enseignant.e.s peut se faire soit de manière directe, soit en interaction avec d'autres instances de socialisation telle que la famille. Plus précisément, nous nous attendons à ce que le niveau d'études des parents influence l'efficacité des interventions des enseignant.e.s.

\section{Méthode}

\subsection{Recrutement et échantillon}

Les données ont été recueillies entre octobre et décembre 2017 auprès d'élèves de collèges et de lycées de la région Provence-Alpes-Côte d'Azur (France). Cette région est le deuxième pôle d'attraction, après la région parisienne, pour les flux migratoires et le nombre de résident.e.s étrangères et étrangers (Institut National de la Statistique et des Études Economiques [INSEE], 2020). La forte attractivité est liée à l'histoire politique locale et aux liens avec les anciennes colonies du Maghreb.

En accord avec les services de la Vie Scolaire des Rectorats, la sélection des établissements s'est faite de manière aléatoire et stratifiée, afin d'interroger des jeunes issu.e.s de milieux socio-économiques et socioculturels hétérogènes. Nous avons sélectionné trois lycées en milieu urbain et périurbain par filière professionnelle, technologique et générale. Dans chaque établissement, la direction a choisi par tirage au sort des élèves de deux classes de seconde, trois classes de première et deux classes de terminale. Quant aux collèges, trois établissements de centre-ville et trois autres de périphérie urbaine ont été choisis. Les directions des établissements ont tiré au sort des élèves de trois classes de quatrième et trois classes de troisième.

L'échantillon $(n=2906)$ se compose de 36,6\% de collégien.ne.s $(n=1063)$ et de $65,4 \%$ de lycéen.ne·s $(n=$ 1843). L'âge moyen est de 13,4 ans pour les collégien-ne.s et 16,2 ans pour les lycéen·ne.s. La répartition de genre est équilibrée à la fois pour le collège (filles 49,5\%) et le lycée (filles 47,0\%).

Conformément à la littérature sur les attitudes intergroupes, nous avons mené nos analyses sur les jeunes appartenant au groupe majoritaire uniquement, définie à partir du lieu de naissance des jeunes et de leurs parents (Baerveld et al., 2004 ; Vermeij et al., 2009). Cette démarche correspond également aux contraintes juridiques que la France impose en matière de collecte de données sur la biographie des individus, où toutes les informations qui peuvent évoquer l'idée d'appartenance à une identité culturelle différente par rapport à la culture républicaine sont bannies de la statistique publique (Simon, 2008). Celles et ceux qui sont né.e.s en France ayant les deux parents nés eux-mêmes en France représentent la population majoritaire $(N=1$ '858), alors que les jeunes né.e.s à l'étranger ou bien né.e.s en France avec au moins un parent né à l'étranger sont codés comme appartenant au groupe minoritaire $\left(\mathrm{N}=1^{\prime} 048\right)$.

\subsection{Variables d'étude}

\subsubsection{Préjugés flagrants et voilés}

Notre variable dépendante est la mesure de l'intolérance envers les groupes minoritaires qui a été étudiée avec les échelles des préjugés flagrants et subtils (Pettigrew et Meertens, 1995, 1997). Ces échelles continuent à faire l'objet de validations dans plusieurs contextes internationaux, encore à nos jours (Civalero et al., 2019 ; Ungaretti et al., 2020) et les critiques quant à la pertinence des deux formes de préjugés se sont désormais effondrées sur le plan méthodologique, étant donné que des différences majeures existent par rapport à la théorie d'origine (voir notamment : Coenders et al., 2001 ; Pettigrew et Meertens, 1993). Les deux échelles sont constituées de dix items chacune, distribués en deux sous-échelles pour les attitudes flagrantes ("menace et rejet », 6 items; « intimité », 4 items) et trois sous-échelles pour les attitudes voilées ("valeurs traditionnelles », 4 items ; " différences culturelles », 4 items ; «sentiments positifs », 2 items). Des exemples d'items pour le préjugé 
flagrant sont les suivants : "La plupart des immigré.e.s sont moins compétent.e.s que les Français.e.s", "La plupart des immigré.e.s occupent des emplois qui devraient revenir aux Français.e.s» ; pour le préjugé voilé : "Beaucoup d'autres groupes sont venus en France, ont surmonté les préjugés et ont réussi. Les immigré.e.s devraient faire de même sans demander un traitement de faveur", "Les immigré.e.s sont différent.e.s des Français.e.s par les valeurs qu'ils et elles enseignent à leurs enfants". Il s'agit d'échelles de type Likert en quatre points pour lesquelles la valeur 1 fait référence à des attitudes positives et la valeur 4 à des attitudes négatives. La cohérence interne du questionnaire (alpha de Cronbach) est de .84 pour le préjugé flagrant et de .76 pour le préjugé voilé. ${ }^{1}$

\subsubsection{Interventions des enseignant $\cdot$ e.s}

Notre variable explicative principale est représentée par les interventions des enseignant.e.s par rapport aux questions posées par la présence des minorités immigrées. Plus précisément, nous avons demandé si les enseignant.e.s, pendant leurs cours, abordaient le thème du racisme et des discriminations. Nous avons aussi interrogé les participant.e.s sur le fait que les us et coutumes des personnes issues de pays étrangers étaient abordés en cours. Pour ce faire, nous avons utilisé les items issus de l'étude de Bekhuis et al. (2012) : Tes enseignant.e.s, pendant leurs cours... a) Abordent-ils.elles le thème du racisme et de la discrimination? ; b) Discutentils.elles des coutumes et des habitudes des personnes issues de pays étrangers? Il s'agit d'items Likert en quatre points allant de 1 (jamais) à 4 (souvent), avec un alpha de Cronbach (.69) acceptable. Les deux items représentent une version adaptée d'échelles déjà utilisées dans des recherches européennes sur l'éducation multiculturelle, notamment aux Pays-Bas (Verkuyten et Thijs, 2002, 2003, 2004).

\subsubsection{Variables de modération}

Afin de vérifier les effets propres à la théorie de la socialisation et saisir certains mécanismes explicatifs qui relient les interventions des enseignant·e.s aux préjugés flagrants et voilés, nous avons mobilisé une variable susceptible de modérer cette relation, à savoir le niveau scolaire des parents : "Quel est le niveau d'études de tes parents? a. primaire, b. brevet de collège, CAP - BEP, c. baccalauréat, brevet technicien.ne supérieur.e, d. études supérieures (licence, master, doctorat) ". La variable a été codée en trois classes : 1=bas (diplôme de collège et BEP/CAP au plus), $2=$ =intermédiaire (baccalauréat et brevet technicien·ne supérieur·e au plus), 3=élevé (licence/master/ doctorat=variable muette).

\subsubsection{Variables de contrôle}

Les variables de contrôle que nous avons mobilisées sont le sexe ("Es-tu un garçon ou une fille? a. garçon, b. fille "; garçon=0, fille=1), le niveau de scolarité (collège=0, lycée=1 ; information administrative fournie par les directions des établissements), la filière d'études au lycée $(0=$ professionnel + technologique, $1=$ général ; information administrative fournie par les directions des établissements), le nombre d'élèves d'origine étrangère inscrit.e.s dans chaque établissement (variable métrique ; information administrative fournie par les directions des établissements) et la confiance exprimée envers le système éducatif (European Values Study, 2008) : "Pourrais-tu me dire dans quelle mesure tu as une grande confiance, une certaine confiance, peu de confiance ou pas confiance du tout dans: a) les enseignant.e.s, b) l'école " (échelle Likert en quatre points allant de $1=$ confiance forte à 4 pas confiance du tout ; alpha de Cronbach.82).

\subsection{Procédure}

Dans chaque établissement l'équipe de recherche a organisé une rencontre avec le groupe d'enseignant·e.s censé.e.s suivre le projet d'enquête où les consignes de présentation et de passation ont été expliquées. Le questionnaire a été administré sous format électronique. Les élèves ont répondu individuellement en salle informatique. Les enseignant.e.s se sont limité.e.s à présenter l'activité et son caractère anonyme. La passation a demandé entre 25 et 40 minutes en fonction de la réactivité de l'élève. Tou·te.s les élèves ont été en mesure

1 L’alpha de Cronbach est utilisé lorsqu'on veut vérifier si les participant.e.s qui ont répondu à deux ou plusieurs questions portant sur des facettes différentes du même concept - dans notre cas, le racisme flagrant ou voilé - ont répondu de manière cohérente aux différents items. Normalement, lorsque les corrélations entre les items d'une échelle correspondent à une valeur alpha non inférieure à 0.6 , il est possible de fusionner les réponses apportées aux différents items dans un seul indice. Dans notre cas, les réponses que chaque participant.e a apportées aux 10 items pour les attitudes de racisme flagrant et aux 10 items pour les attitudes de racisme voilé ont été fusionnées dans deux indices distincts. Plus le score de chaque indice est élevé, plus intense est le sentiment d'intolérance raciste flagrante ou voilée. 
de comprendre et de répondre au questionnaire, préalablement validé par le Comité National Informatique et Libertés. Chaque établissement avait informé les parents des élèves au sujet de cette passation (aucun refus n'a été communiqué aux équipes de direction). Lors de la passation, les élèves avaient le droit de quitter la salle à tout moment, mais aucun élève n’a refusé de compléter le questionnaire.

\subsection{Analyse des données}

Plusieurs étapes ont été prévues. Dans un premier temps, nous avons vérifié la cohérence de la structure factorielle des échelles pour la mesure des préjugés flagrants et voilés. Ensuite, nous avons mené une analyse descriptive pour identifier si les variables sociales et scolaires influencent la perception des interventions des enseignant.e.s de la part des élèves. Dans un second temps, au moyen d'analyses de régression linéaire multiple, nous avons vérifié l'effet de ces interventions sur les manifestations d'intolérance des élèves. Enfin, nous avons mobilisé une analyse de modération pour étudier les effets d'interaction entre les interventions des enseignant.e.s perçues par les élèves, leurs préjugés et le capital scolaire de leurs familles.

Les analyses ont été menées par le logiciel SPSS 24 intégré par la version 3.0 de Process (C A. E. Hayes 2018) pour les analyses de médiation et de modération.

\section{Résultats}

\subsection{La perception de L'investissement des enseignant-e-s et ses effets sur Les préjugés des élèves}

Afin d'être sûr·e.s que les élèves aient saisi la différence entre les deux concepts (préjugés flagrants et voilés) et les sous-dimensions qui les composent, nous avons mené au préalable une analyse factorielle exploratrice (ACP - rotation varimax). Les résultats confirment que la structure factorielle des deux échelles de racisme flagrant et voilé est identique à celle qui a été identifiée par Pettigrew et Meertens (1995, 1997). Pour l'échelle sur les attitudes flagrantes, deux composantes expliquent 69\% de la variance totale et correspondent aux deux sous-échelles de " menace et rejet " et " refus intimité ». En ce qui concerne les attitudes de racisme voilé, trois composantes expliquent $66 \%$ de la variance totale et correspondent aux sous-échelles "valeurs traditionnelles ", "différences culturelles » et "sentiments positifs ». Le fait d'avoir retrouvé les mêmes sous-échelles que celles de la théorie d'origine confirme la pertinence des outils de mesure, y compris pour une population d'adolescent.e.s pour laquelle rares sont les études (voir par ex. Sarafidou et al., 2013).

Le tableau 1 présente les statistiques descriptives des échelles de racisme flagrant et voilé ainsi que de notre principale variable explicative, à savoir les interventions des enseignant.e.s sur les questions en lien avec le racisme, les discriminations et la diversité.

\section{Tableau 1}

Variables dépendantes et indépendantes - statistiques descriptives

\begin{tabular}{c|c|c|c|c|c}
\hline \multicolumn{3}{c|}{ Variables dépendantes } & \multicolumn{2}{c}{ Variable indépendante } \\
\hline \multicolumn{2}{c|}{ Racisme flagrant } & \multicolumn{2}{c|}{ Racisme voilé } & \multicolumn{2}{c}{ Interventions des enseignant.e.s } \\
\hline $\boldsymbol{M}$ & $\boldsymbol{S D}$ & $\boldsymbol{M}$ & $\boldsymbol{S D}$ & $\boldsymbol{M}$ & $\boldsymbol{S D}$ \\
\hline 18,0 & 5,9 & 24,9 & 5,8 & 4,5 & 1,5 \\
\hline
\end{tabular}

Dans un premier temps, nous avons vérifié si les variables dépendantes et la variable explicative étaient reliées. La présence de corrélations significatives entre les deux types de préjugés et les interventions des enseignant.e.s (tableau 2) confirme nos hypothèses théoriques qui postulent des relations entre les interventions perçues par les élèves et leurs préjugés flagrants et voilés. 
Tableau 2

Corrélations entre variables dépendantes et indépendantes (Pearson)

\begin{tabular}{|c|c|c|c|c|}
\hline & & Racisme flagrant & Racisme voilé & $\begin{array}{c}\text { Interventions des } \\
\text { enseignant.e.s }\end{array}$ \\
\hline \multirow[t]{2}{*}{ Racisme flagrant } & Corrélation de Pearson & 1 &, $628^{* *}$ &,$- 166^{* *}$ \\
\hline & $p$ & &, 000 &, 001 \\
\hline \multirow[t]{2}{*}{ Racisme voilé } & Corrélation de Pearson &, $628^{* *}$ & 1 &,$- 114^{* *}$ \\
\hline & $p$ &, 000 & &, 081 \\
\hline \multirow[t]{2}{*}{ Interventions des enseignant.e.s } & Corrélation de Pearson &,$- 166^{* *}$ &,$- 114^{* *}$ & 1 \\
\hline & $p$. &, 001 &, 081 & \\
\hline
\end{tabular}

** La corrélation est significative au niveau 0,01 (bilatéral)

Pour ce qui a trait à la première hypothèse de l'étude, à savoir la relation entre les interventions des enseignant·e.s perçues par les élèves, le profil social de ces dernières et derniers et les caractéristiques de leurs parcours scolaires, les résultats de l'ANOVA révèlent que l'investissement des enseignant.e.s quant aux questions en lien avec le racisme, les discriminations et la diversité est perçu davantage par les filles par rapport aux garçons ( $\mathrm{p} \leq .001-\mathrm{F}$ 36,5). Quant aux effets du parcours scolaire, la seule variable significative est le type de filière lycéenne : l'investissement des enseignant.e.s est davantage perçu par les élèves des filières technologiques et professionnelles par rapport aux élèves des filières générales ( $\mathrm{p} \leq .002$ - F 9,9). On pouvait s'attendre à ce que la vigilance des élèves soit plus marquée chez les jeunes issu.e.s de familles avec un capital scolaire plus important et qui ont plus souvent de meilleurs résultats scolaires, ce qui pourrait prédire une plus grande attention et vigilance par rapport aux pratiques des leurs enseignant.e.s (dans le cadre de cette étude la corrélation entre le niveau de diplôme des parents et le rendement scolaire est égale à .48, Pearson). En revanche, le niveau d'études des parents n'a aucun effet significatif sur la perception de l'investissement des enseignant.e.s. En ce qui concerne les variables scolaires, on s'attendait à ce que la perception de l'investissement des enseignant.e.s ait été plus importante pour les lycéen·ne.s par rapport aux collégien·ne·s, alors que le niveau d'études n'exerce aucun effet significatif sur la perception des élèves.

Pour vérifier les effets directs entre les interventions des enseignant·e.s perçues par les élèves et les préjugés de ces dernières et derniers, nous avons mené des analyses de régression linéaire multiple (méthode " Entrée ") sur l'échantillon d'élèves d'origine majoritaire. Les résultats pour les préjugés flagrants sont présentés dans le tableau 3.

Tableau 3

\begin{tabular}{|c|c|c|c|c|}
\hline & & Erreur standard & Bêta & Sig \\
\hline \multirow{7}{*}{$\begin{array}{l}\text { Modèle } 1 \\
N 2226\end{array}$} & (Constante) & ,767 & & \\
\hline & Sexe*** & ,305 &,- 122 & .000 \\
\hline & $\begin{array}{l}\text { Niveau étude parents } \\
\text { Niveau bas } v \text { élevée** } \\
\text { Niveau intermédiaire } v s \\
\text { élevé*** }\end{array}$ & $\begin{array}{l}\text {,363 } \\
380\end{array}$ & $\begin{array}{l}, 113 \\
, 134\end{array}$ & $\begin{array}{l}.000 \\
.000\end{array}$ \\
\hline & Niveau école* & 391 &,- 058 & .036 \\
\hline & Filière d'études au lycée & 307 & ,023 & .416 \\
\hline & Confiance système éducatif** & ,092 &,- 088 & .001 \\
\hline & Total élèves étrangers** & 003 & 083 & .002 \\
\hline \multirow{8}{*}{$\begin{array}{l}\text { Modèle } 2 \\
\text { N } 2225\end{array}$} & (Constante) & .876 & & \\
\hline & Sexe $e^{* * *}$ & 307 &,- 114 & .000 \\
\hline & $\begin{array}{l}\text { Niveau étude parents } \\
\text { Niveau bas vs élevée** } \\
\text { Niveau intermédiaire } v s \\
\text { Élevé*** }\end{array}$ & $\begin{array}{l}363 \\
379,\end{array}$ & $\begin{array}{l}, 113 \\
, 135\end{array}$ & $\begin{array}{l}.000 \\
.000\end{array}$ \\
\hline & Niveau école* & 390 &,- 055 & .045 \\
\hline & Filière d'études au lycée & ,307 &, 021 & .416 \\
\hline & Confiance système éducatif** & 092 &,- 086 & .001 \\
\hline & Total élèves étrangers** & 003 & ,086 & .001 \\
\hline & Interventions des enseignant.e.s** &, 100 &,- 065 & .010 \\
\hline
\end{tabular}

${ }^{* * *} \mathrm{p} \leq .001,{ }^{* *} \mathrm{p} \leq .01,{ }^{*} \mathrm{p} \leq .05$ 
Pour le groupe de jeunes majoritaires, les préjugés flagrants sont plus prononcés chez les garçons que les filles ( $\mathrm{p} \leq .001$ ), chez les élèves dont les parents ont des niveaux d'étude bas ( $\mathrm{p} \leq .001)$ et intermédiaires ( $\mathrm{p} \leq .001)$ par rapport aux élèves dont les parents ont effectué des études supérieures. En ce qui concerne le niveau scolaire, ce sont les collégien-ne.s qui se démarquent par des attitudes plus flagrantes par rapport aux lycéen·ne.s ( $\mathrm{p} \leq .051)$. Elles s'intensifient au fur et mesure que la confiance envers le système éducatif baisse ( $\mathrm{p} \leq .001)$. Enfin, plus l'établissement accueille des élèves d'origine étrangère, plus l'intolérance flagrante est élevée ( $\mathrm{p} \leq .001)$. L'ajout de la variable sur l'action des enseignant.e.s améliore de manière significative la prédictivité du modèle $\left(\mathrm{R}^{2} .22\right.$ $\mathrm{p}$ 5.001). Plus les élèves déclarent que les enseignant.e.s abordent les questions liées au racisme, aux discriminations, à la diversité, plus les préjugés flagrants baissent $(\mathrm{p} \leq .01)$. La filière d'études au lycée ne montre aucun effet significatif.

Le tableau 4 présente les résultats pour les préjugés voilés.

\section{Tableau 4}

Régression linéaire multiple (méthode "entrez») - Racisme voilé

\begin{tabular}{|c|c|c|c|c|}
\hline & & Erreur standard & Bêta & Sig. \\
\hline \multirow{7}{*}{$\begin{array}{l}\text { Modèle } 1 \\
N 2143\end{array}$} & (Constante) & ,781 & & .000 \\
\hline & Sexe $e^{* * *}$ &, 311 &,- 082 & .000 \\
\hline & $\begin{array}{l}\text { Niveau étude parents } \\
\text { Niveau bas vs élevée** } \\
\text { Niveau intermédiaire } v s \\
\text { élevé }^{* * *}\end{array}$ & $\begin{array}{l}, 369 \\
, 386\end{array}$ & $\begin{array}{l}, 115 \\
, 095\end{array}$ & $\begin{array}{l}.000 \\
.000\end{array}$ \\
\hline & Niveau école* & ,391 & 007 & .036 \\
\hline & Filière d'études au lycée & ,312 &, 017 & .371 \\
\hline & Confiance système éducatif** & 094 &,- 080 & .001 \\
\hline & Total élèves étrangers** &, 003 &, 083 & .002 \\
\hline \multirow{8}{*}{$\begin{array}{l}\text { Modèle } 2 \\
\text { N } 2143\end{array}$} & (Constante) & ,844 & & .000 \\
\hline & Sexe $e^{* * *}$ & 312 &,- 076 & .000 \\
\hline & $\begin{array}{l}\text { Niveau étude parents } \\
\text { Niveau bas } v \text { s élevé*** } \\
\text { Niveau intermédiaire } v s \\
\text { élevée** }\end{array}$ & $\begin{array}{l}, 368 \\
, 386\end{array}$ & $\begin{array}{l}, 114 \\
, 094\end{array}$ & $\begin{array}{l}.000 \\
.000\end{array}$ \\
\hline & Niveau école* & ,390 &, 007 & .045 \\
\hline & Filière d'études au lycée & ,312 &, 015 & .416 \\
\hline & Confiance système éducatif** & ,094 &,- 078 & .001 \\
\hline & Total élèves étrangers** & 003 & 083 & .001 \\
\hline & Interventions des enseignant $\cdot e \cdot s^{*}$ & , 177 &,- 065 & .035 \\
\hline
\end{tabular}

${ }^{* * *} \mathrm{p} \leq .001,{ }^{* *} \mathrm{p} \leq .01,{ }^{*} \mathrm{p} \leq .05$

Les manifestations de racisme voilé sont principalement prégnantes chez les garçons par rapport aux filles ( $\mathrm{p}$ $\leq .001)$, les jeunes ayant des parents avec des parcours scolaires courts ( $\mathrm{p} \leq .001)$ ou intermédiaires ( $\mathrm{p} \leq .001)$ par rapport aux jeunes dont les parents ont suivi des études supérieures. Ensuite, la force des préjugés voilés est inversement proportionnelle à la confiance que l'on manifeste envers le système éducatif ( $\mathrm{p} \leq .01)$. Enfin, plus l'établissement accueille d'élèves d'origine étrangère, plus les attitudes d'intolérance voilée sont marquées ( $\mathrm{p}$ s.01). L'ajout de la variable concernant les interventions des enseignant.e.s accroit la prédictibilité du modèle $\left(\mathrm{R}^{2} 19 \mathrm{p} \leq .01\right)$. Bien que l'effet soit moins important par rapport aux effets sur les préjugés flagrants, plus les élèves ont le sentiment que les enseignant.e.s abordent les questions liées au racisme, aux discriminations et à la diversité, plus les préjugés voilés baissent ( $\mathrm{p} \leq .05)$. La filière d'études au lycée et le niveau école ne montrent aucun effet significatif.

La principale hypothèse de l'étude est confirmée : plus les élèves ont le sentiment que leurs enseignant.e.s abordent en cours les questions en lien le racisme, les discriminations et la diversité, plus leurs préjugés flagrants et voilés envers les minorités immigrées baissent. 


\subsection{Les effets de la socialisation familiale}

Les effets directs de la perception de l'investissement des enseignant·e.s sur les préjugés flagrants et voilés des élèves ont été étudiés par le biais d'autres facteurs, tel que le capital scolaire des parents. Pour vérifier si le niveau d'études des parents exerce une influence sur l'efficacité des interventions des enseignant.e.s perçues par les élèves, nous avons mené une analyse de modération dans laquelle le niveau d'études des parents était la variable modératrice $(\mathrm{W})$. Ce type d'analyse permet de vérifier si une première relation directe et significative entre une variable indépendante $(\mathrm{X})$ et une variable dépendante $(\mathrm{Y})$ n'est pas affectée par l'intervention d'une troisième variable $(\mathrm{W})$, qui peut accroitre ou bien affaiblir la force de la relation initiale.

Concernant les préjugés flagrants, l'effet du modérateur est compris dans l'intervalle de confiance (int. -.0706) : la variable $(\mathrm{W})$ interagit sur la variable $(\mathrm{X})(\alpha 3=\leq .05)$ pour influencer $(\mathrm{Y})(\alpha 2=.005 \neq 0)$. Le fait d'avoir des parents qui ont suivi des études supérieures $(\alpha-.49)$ représente une condition qui renforce les effets des interventions des enseignant.e.s par rapport aux élèves dont les parents ont suivi des scolarités intermédiaires $(\alpha-.42)$ ou courtes $(\alpha-.34)$. Le cadre d'analyse pour les manifestations d'intolérance voilées est similaire à celui de l'intolérance flagrante. Le modèle est significatif, avec un effet modérateur compris dans l'intervalle de confiance (int. -.0357) : la variable (W) interagit sur la variable $(\mathrm{X})\left(\alpha_{3}=\leq .05\right)$ pour influencer $(\mathrm{Y})\left(\alpha_{2}=.005\right.$ $\neq 0)$. Les interventions des enseignant.e.s contribuent à la diminution des préjugés voilés lorsque les parents ont effectué des études supérieures $(\alpha-.41)$ par rapport aux élèves dont les parents ont suivi des scolarités intermédiaires $(\alpha-.37)$ ou courtes $(\alpha-.33)$.

La deuxième hypothèse de l'étude est aussi confirmée : un niveau d'études élevé des parents (niveau supérieur) augmente l'efficacité des interventions des enseignant.e.s sur le niveau de préjugés racistes flagrants et voilés chez les élèves.

\section{Discussion et conclusion}

L'étude montre que plus les élèves ont le sentiment que les enseignant·e.s abordent les questions du racisme, des discriminations et de la diversité, plus les préjugés racistes flagrants et voilés diminuent. Il est vrai que nous ne disposons pas d'information quant à la nature de ces interventions, leurs contenus et les modalités déployées par les enseignant.e.s afin de les discuter en classe. Une démarche qualitative serait envisageable pour recueillir ces compléments d'information et mieux comprendre les réactions des élèves lorsqu'ils et elles sont confronté.e.s à ce type d'interventions. Cela permettrait notamment de mieux cerner si ces réactions génèrent des répercussions sur l'engagement des enseignant.e.s, ainsi que d'appréhender davantage la posture des directions d'établissements envers la diversité culturelle. Comme déjà indiqué, notre étude a pointé une tendance plus générale qui a trait à la relation enseignant·e-s-élèves, sans pouvoir apporter d'informations quant au contexte et à la pédagogie mise en œuvre.

Nos résultats en matière de baisse des préjugés chez les élèves laissent entendre qu'il s'agit bien d'interventions qui visent à déconstruire les sentiments de peur et de menace qui souvent enferment les minorités immigrées dans des représentations négatives. C'est pour ces raisons qu'un sujet comme celui que nous avons traité dans cette étude mériterait également d'être analysé au moyen d'une démarche plus expérimentale, dans laquelle les enseignant·e.s seraient formé·e.s quant aux stratégies de sensibilisation aux questions citées. Des mesures pré-test et post-test des attitudes des élèves pourraient nous renseigner quant à l'efficacité de l'intervention. Toutefois, il serait impossible de contrôler toutes les sources de variation - par exemple, les réactions des élèves et les répercussions sur les interventions des enseignant.e.s, leur capacité à s'accommoder aux exigences de normativité sociale sans que cela altère leurs convictions de départ.

Pour revenir à notre étude, un point à retenir est que lorsque l'engagement des enseignant.e.s est tangible aux yeux des élèves, celui-ci contribue à la diminution des préjugés flagrants et voilés. Comme suggéré par Bekhuis et al. (2012), plus les systèmes éducatifs se font les porte-paroles de valeurs de tolérance et de respect de l'Autre, plus le rôle joué par le corps enseignant est important. Dans la même direction, Verkuyten et Kinket (2000), ainsi que Verkuyten et Thijs (2000) soulignent que le fait d'aborder des questions en lien avec les minorités immigrées permet de briser certains tabous et, par exemple, de dénoncer plus facilement les manifestations de racisme et les discriminations que les élèves peuvent subir au sein de l'espace scolaire. Ainsi, non seulement l'intervention a un effet certain sur les préjugés des élèves mais elle permet aussi de contraster la loi du silence à laquelle sont soumises bien des victimes (Debarbieux, 1998).

Nos premières analyses descriptives indiquent qu’il existe un véritable « effet enseignant ». Parmi les variables mobilisées pour analyser les perceptions des élèves, le genre, l'origine et le type de filière lycéenne exercent des 
effets significatifs. La plus grande attention des filles à la fréquence des interventions des enseignant.e.s par rapport aux garçons s'inscrit probablement dans les effets propres à la socialisation de genre sur la scolarité, les filles ayant intériorisé plus solidement des attitudes d'écoute et d'exécution (Arnold et Candea, 2015). La perception accrue des élèves des filières technologiques et professionnelles par rapport aux élèves des filières générales est probablement en lien avec les caractéristiques socio-démographiques de ces parcours. Il s'agit d'établissements qui ont une probabilité plus élevée d'accueillir des populations d'élèves issus des classes moyennes ou défavorisées et parmi lesquelles la proportion d'élèves issus des minorités immigrées est certainement plus importante par rapport aux filières littéraires et scientifiques. Confronté.e.s à une plus grande mixité sociale, les enseignant.e.s seraient plus motivé.e.s à aborder les questions en lien avec l'immigration.

Nous avons pu vérifier que la relation entre le capital scolaire de la famille et les attitudes racistes des jeunes n'est pas aussi linéaire que le montrent d'autres études (Schuman et al., 1997). Nos résultats ont mis en exergue que le niveau de préjugé des jeunes est lié aux ressources scolaires des parents : les élèves de familles démunies en ressources scolaires font preuve d'une intolérance plus forte que les élèves dont les parents ont effectué des études longues. Le capital scolaire étant relié à la position dans le marché de l'emploi, il est possible que les parcours scolaires courts fassent ressentir un plus fort sentiment de compétition envers les minorités immigrées, ces dernières étant surreprésentées dans les strates les plus basses du marché de l'emploi. En ce qui concerne ses effets sur les interventions des enseignant.e.s perçues par les élèves, le capital scolaire des parents intervient seulement de manière indirecte en tant que variable qui en renforce l'efficacité, alors qu'il n'exerce pas un effet direct sur le niveau d'investissement perçu par les élèves. Il s'agit d'une différence subtile mais qui nous livre un cadre aux contours bien précis. Le bagage scolaire de la famille pourrait contribuer à une plus grande efficacité des actions des enseignant.e.s, car si le capital scolaire familial des plus instruit.e.s permet potentiellement de déconstruire plus aisément les stéréotypes (Hagendoorn et Nekuee, 1999), il peut aussi mobiliser les ressources pour maquiller plus facilement des attitudes ou opinions racistes, intolérantes ou xénophobes. Il s'agit de la dynamique sous-jacente aux manifestations flagrantes ou voilées des préjugés envers les minorités ethniques. Cette dynamique est le résultat de l'interaction entre les ressources personnelles de chacun.e et les caractéristiques liées à l'arrière-plan social des individus. De faibles ressources scolaires et culturelles réduisent les moyens intellectuels pour neutraliser les sentiments d'hostilité et être capables de prendre de la distance. En revanche, lorsque le niveau d'étude est plus élevé, le recours aux préjugés voilés est plus fréquent, probablement en raison de capacités d'élaboration rhétorique plus importantes. Dans les deux cas, il s'agit de situations sociales où le regard des autres est majoritairement celui des pair.e.s, autrement dit des individus avec qui on partage un profil sociologique similaire (Bergamaschi et Santagati, 2019).

Notre recherche souligne la complexité des effets que l'éducation peut exercer sur les préjugés racistes, flagrants ou voilés. Nous avons bien sûr des effets linéaires mais ils s'articulent de manière complexe selon le profil des élèves. Nos résultats montrent que les enseignant·e.s ont leur mot à dire et, notamment, un rôle à jouer qui est loin d'être anodin sur la diffusion des idées intolérantes.

L'importance de ces aspects doit être appréhendée à la lumière du type de population que nous avons étudié : il s'agit d'adolescent.e.s qui commencent à se faire une idée des principes d'égalité, de justice et des rapports au sein de la société. Cela signifie que la socialisation n'amène pas à la simple intériorisation de normes, codes et valeurs. Elle permet également l'appropriation de l'ensemble des rapports de pouvoir, des répertoires normatifs et symboliques qui permettent de nommer l'Autre et qui représentent l'environnement où l'individu se trouve dans les différents moments de la vie. Nous rejoignons Sniderman et Hagendoorn (2007) lorsqu'ils soulignent que la réduction des préjugés par l'éducation est une thèse qui nécessite beaucoup de précautions et qu'elle mérite d'être davantage explorée.

\section{Références bibliographiques}

Arnold, A., et Candea, M. (2015). Comment étudier l'influence des stéréotypes de genre et de race sur la perception de la parole? Langage et société, 152(2), 75-96. https://doi.org/10.3917/1s.152.0075

Baerveldt C., Van Dujing M., Vermeij L., et Dianne A. V. H. (2004). Ethnic boundaries and personal choice. Assessing the influence of individual inclinations to choose intra-ethnic relationships on pupils' networks. Social Networks-26(1), 55-74. https://doi.org/10.1016/j.socnet.2004.01.003

Banks, J. A. (Dir.). (1996). Multicultural Education, Transformative Knowledge and Action. Teachers College Press.

Barker, M. (1984). The New Racism: Conservatives and the Ideology of the Tribe. Aletheia Press.

Bekhuis, H., Ruiter, S., et Coenders, M. (2012). Xenophobia among youngsters: The effect of inter-ethnic contact. European Sociological Review, 29(2), 229-242. https://doi.org/10.1093/esr/jcr057

Bell, D. (1992). Race, Racism and American Law. Little Brown. 
Bergamaschi, A., et Santagati, M. (2019). When friendship is stronger than prejudice. Role of intergroup friendships among adolescents in two distinct socio-cultural contexts of immigration. International Review of Sociology, 29(1), 36-57. https://doi. org/10.1080/03906701.2019.1609750

Bonilla-Silva, E. (1997). Rethinking Racism: Toward a structural interpretation. American Sociological Review, 62(3), 465-480. https://doi.org/10.2307/2657316

Bonnery, S. (2006). La question de «l'ethnicité» dans l'École: essai de reconstruction du problème. Société et jeunesses en difficultés, $1,1-17$.

Brinbaum, Y., et Primon, J.-L. (2016). Parcours scolaires des descendants d'immigrés et sentiments d'injustice et de discrimination. Économie et statistique, 464, 465-466.

Brown, B. A., et Mutegi, J. W. (2010). A paradigm of contradictions: Racism and Science Education. Dans International Encyclopédia of Education (p. 554-564). Elsevier.

Civalero, L., Alonso, D., et Brussino, S. (2019). Evaluation of the prejudice towards immigrants: Argentine adaptation of the scale of subtle and blatant prejudice. Ciencias Psicológicas, 13(1), 119-133.

Coenders, M., et Scheepers, P. (2003). The effect of education on nationalism and ethnic exclusionism. Political Psychology, 24(2), 313-343. https://doi.org/10.1111/0162-895X.00330

Coenders, M., Scheepers, P., Sniderman, P. M., et Verberk, G. (2001). Blatant and subtle prejudice: Dimensions, determinants, and consequences; Some comments on Pettigrew and Meertens. European Journal of Social Psychology, 31(3), 281-297. https:// doi.org/10.1002/ejsp.44

De Rudder V., Poiret, C., et Vourc'h, F. (2000). L'inégalité raciste. L'universalité républicaine à l'épreuve. Presses Universitaires de France.

Debarbieux, E. (1998). Violence à l'école, un défi mondial? Armand Colin.

Duru-Bellat, M., et van Zanten, M. (2012). Sociologie de l'école. Armand-Colin.

European Network Against Racism. (2015-2016). Racism and Discrimination in the Context of Migration in Europe. European Commission.

European Values Study. (2008). Survey 2008. https://europeanvaluesstudy.eu/methodologydata-documentation/previoussurveys-1981-2008/survey-2008/

Gaertner, S., et Dovidio, J. (1984). Prejudice, Discrimination and Racism. Academic Press.

Geerlings, J., Thijs, J., et Verkuyten, M. (2017). Student-teacher relationships and ethnic outgroup attitudes among majority students. Journal of Applied Developmental Psychology, 52, 69-79. https://doi.org/10.1016/j.appdev.2017.07.002

Grosland, T. J. (2019). Through laughter and through tears: Emotional narratives to antiracist pedagogy. Race Ethnicity and Education, 22(3), 301-318. https://doi.org/10.1080/13613324.2018.1468750

Haegel, F. (1999). The effect of education on the expression of negative views towards immigrants in France: The influence of the republican model put to the test. Dans L. Hegendoorn et S. Nekeuee (Dir.), Education and Racism. A cross national inventory of positive effects of education on ethnic tolerance (p. 33-46). Pallgrave.

Hagendoorn, L., et Nekuee, S. (1999). Education and Racism. A cross national inventory of positive effects of education on ethnic tolerance. Palgrave.

Hayes, A. F. (2018). Introduction to mediation, moderation, and conditional process analysis (2e éd.). The Guilford Press.

Hello, E., Scheepers, P., et Gijsberts, M. (2010). Education and ethnic prejudice in Europe. Explanations for cross-national variances in the educational effect on ethnic prejudice. Scandinavian Journal of Educational Research, 46(1), 5-24. https://doi. org/10.1080/00313830120115589

Hyman, H. H., et Wright, C. R. (1979). Education's Lasting Effect on Values. University of Chicago Press.

Institut National de la Statistique et des Etudes Economiques. (2020). L'essentiel sur... les immigrés et les étrangers. https://www. insee.fr/fr/statistiques/3633212

Lahire, B. (2017). Sociological biography and socialisation process: A dispositionalist-contextualist conception. Contemporary Social Sciences, 2, 1-15. https://doi.org/10.1080/21582041.2017.1399213

Miller, K. A., Kohn, M. J., et Schooler, C. (1985). Educational self-direction and the cognitive functioning of students. Social Forces, 63(4), 923-944. https://doi.org/10.1093/sf/63.4.923

Moguérou, L., Brinbaum Y., L., et Primon, J.-L. (2015). Les ressources scolaires des immigrés à la croisée des histoires migratoires et familiales. Dans C. Beauchemin, C. Hamel, et P. Simon (Dir.), Trajectoires et origines. Enquête sur la diversité des populations en France (p. 147-174). Éditions de l'INED.

Ostinelli, M. (2020). La legittimità dell'educazione alla cittadinanza. Questioni filosofiche. Revue Suisse des Sciences de l'Education, 42(1), 23-45.

Ouellet, F. (2002). Léducation interculturelle et l'éducation à la citoyenneté. Quelques pistes pour s'orienter dans la diversité des conceptions. VEI enjeux, 129, 146-167.

Pagé, M. (2001). L'éducation à la citoyenneté devant la diversité des conceptions de la citoyenneté. Dans M. Pagé, F. Ouellet, et L. Cortesao (Dir.), L'Éducation à la citoyenneté. Editions du CRP.

Pérez, R. (2013). Learning to make racism funny in the 'color-blind'era: Stand-up comedy students, performance strategies, and the (re) production of racist jokes in public. Discourse et Society, 24(4), 478-503. https://doi.org/10.1177/0957926513482066

Perroton, J. (2000). Les dimensions ethniques de l'expérience scolaire. L'Année sociologique, 50(2), 437-467.

Pettigrew, T. F., et Meertens, R. W. (1993). Le racisme voilé: dimensions et mesure. Dans M. Wieviorka (Dir.), Racisme et modernité (p. 109-126). La Découverte.

Pettigrew, T. W.,et Meertens, R. W. (1995). Subtle and blatant prejudice in western Europe. European Journal of Social Psychology, 25(1), 57-75. https://doi.org/10.1002/ejsp.2420250106

Pettigrew, T. W., et Meertens, R. W. (1997). Is subtle prejudice really prejudice? Public Opinion Quarterly, 61, 54-71. https://www. jstor.org/stable/2749511 
Roussier-Fusco, E. (2003). Le modèle français d'intégration et les dynamiques interethniques dans deux écoles de ta banlieue parisienne. Revue Française de Pédagogie, 144, 29-37.

Sanchez-Mazas, M., et Fernandez-Iglesias, R. (2002). L'interculturel à l'épreuve de l'action : comment équiper les enseignants face au public scolaire hétérogène ? Alterstice, 1(1), 35-46.

Sarafidou, J-A., Govarist, C., et Loumakou, M. (2013). The subtle-blatant distinction of ethnic prejudice among ethnic majority chidren. Intercultural Education, 24(3), 264-276. https://doi.org/10.1080/14675986.2013.799805

Scheepers, P., Gijsberts, M., et Hello, E. (2002). Religiosity and prejudice against ethnic minorities in Europe: Cross-national tests on a controversial relationship. Review of Religious Research, 43(3), 242-265. https://doi.org/10.2307/3512331

Schuman, H., Steeh, C., Bobo, L., et Krysan, M. (1997). Racial Attitudes in America: Trends and Interpretations. Harvard University Press.

Selznick, G. J., et Steinberg, S. (1969). The tenacity of prejudice. Anti-Semitism in contemporary America. Harper and Row.

Simon, P. (2008). Les statistiques, les sciences sociales françaises et les rapports sociaux ethniques et de " race ". Revue Française de Sociologie, 49(1), 153-162. https://doi.org/10.3917/rfs.491.0153

Sniderman, P. M., et Hagendoorn, L. (2007). When Ways of Life Collide. Princeton University Press.

Ungaretti, J., Edgardo, E., et Barreiro, A. (2020). Validation of the subtle and blatant prejudice scale towards indigenous people in Argentina. Current Psychology, 39, 1423-1429. https://doi.org/10.1007/s12144-018-9844-4

Varvaet, R., Van Houtte, M., et Stevens, P-A., J. (2018). Multicultural school leadership, multicultural teacher culture and the ethnic prejudice of Flemish pupils. Teaching and Teacher Education, 76, 68-77. https://doi.org/10.1016/j.tate.2018.08.009

Verkuyten, M., et Kinket, B. (2000). Social distances in a multi-ethnic society: The ethnic hierarchy among Dutch pre-adolescents. Social Psychology Quarterly, 63(1), 75-85. https://doi.org/10.2307/2695882

Verkuyten, M., et Thijs, J. (2000). Leren (en) Waarderen: Discriminatie, zelfbeeld, relaties en leerprestaties in witte en zwarte basisscholen. Thela Thesis.

Verkuyten, M., et Thijs, J. (2002). Multiculturalism among minority and majority adolescents in the Netherlands. International Journal of Intercultural Relations, 26, 91-108. https://doi.org/10.1016/S0147-1767(01)00039-6

Verkuyten, M., et Thijs, J. (2003). Racist victimization among children in The Netherlands: The effect of ethnic group and school. Ethnic and Racial Studies, 25(2), 310-331. https://doi.org/10.1080/01419870120109502

Verkuyten, M., et Thijs, J. (2004). Global and ethnic selfesteem in school context: Minority and majority groups in the Netherlands. Social Indicators Research, 67, 253-281. https://doi.org/10.1023/B:SOCI.0000032339.86520.5f

Verkuyten, M., et Thijs, J. (2013). Multicultural Education and inter-ethnic attitudes. An intergroup perspective. European Psychologist, 18(3), 179-190. https://doi.org/10.1027/1016-9040/a000152.

Vermeij, L., Marijtje, M., et Baerveldt, C. (2009). Ethnic segregation in context: Social discrimination among native Dutch pupils and their ethnic minority classmates. Social Networks, 31, 230-239. https://doi.org/10.1016/j.socnet.2009.06.002

Vogt, W. P. (1997). Tolerance and Education. Sage Publications.

Mots-clés : Préjugé ; système éducatif ; élève ; structure sociale ; tolérance

\section{Schülerwahrnehmungen zu offenen und verdeckten Formen der Vorurteilsprävention: Wenn Lehrerlnnen zu Wort kommen}

\section{Zusammenfassung}

Dieser Artikel untersucht die «offenen» und «verdeckten» Vorurteile, die von 1'858 Schülern der Oberstufe und Gymnasium in Frankreich mittels einer Fragebogenerhebung geäussert wurden. Eine erste deskriptive Analyse ermöglicht es, die sozialen und schulischen Faktoren zu identifizieren, die die Wahrnehmungen der Schüler über das Engagement der Lehrer und Lehrerinnen einwanderungsbezogenen Themen beeinflussen können. Multivariate Analysen zeigen, dass intolerante Einstellungen abnehmen, wenn LehrerInnen mit ihren Schülern über Themen, die im Zusammenhang mit Rassismus stehen, wie Diskriminierung und kulturelle Unterschiede, sprechen. Mediations- und Moderationsanalysen weisen auf externe Faktoren hin, welche die Interventionen der Lehrer und Lehrerinnen für die Schüler und Schülerinnen angemessen erscheinen lassen.

Schlagworte: Vorurteile; Bildungssystem; SchülerInnen; Sozialstruktur; Toleranz 


\section{La prevenzione dei pregiudizi «manifesti» e «sottili» percepita dagli allievi: quando le parole degli insegnanti contano}

Riassunto

Questo articolo si focalizza sui pregiudizi " manifesti " e "sottili " espressi da 1.858 allievi delle scuole medie e superiori in Francia mediante un'indagine quantitativa. Una prima analisi descrittiva permette di identificare i fattori sociali e scolastici che possono influenzare la percezione degli studenti del coinvolgimento degli insegnanti nei problemi legati all'immigrazione. Utilizzando modelli multivariati, i risultati evidenziano che quando gli studenti dichiarano che i loro insegnanti affrontano questioni legate al razzismo, alla discriminazione e alla diversità culturale il livello di intolleranza nei confronti delle minoranze etniche diminuisce. Inoltre, un'analisi causale mette in luce l'esistenza di fattori esterni che facilitano l'appropriazione degli interventi degli insegnanti.

Parole chiave: Pregiudizio; sistema scolastico; allievi, struttura sociale; tolleranza

\section{Prevention of blatant and subtle prejudices as perceived by students: when teachers have their say}

Summary

This article focuses on "blatant" and "subtle" prejudices expressed by 1858 lower and upper secondary school students in France, in a self-reported survey. A first descriptive analysis allows to identify the social and school factors that may influence students' perception of teachers' involvement regarding issues raised by migration. Multivariate analyses show that when students state that their teachers address issues related to racism, discrimination and cultural diversity, intolerant attitudes decrease. Mediation and moderation models highlight the existence of external factors facilitating the appropriation of teachers' interventions by students.

Keywords: Prejudice; educational system; student; social structure; tolerance 
Alessandro Bergamaschi est maître de conférences en sociologie de l'éducation à l'Université Côte d'Azur. À l'intersection des paradigmes critiques et institutionnalistes, ses travaux de recherche portent, d'une part, sur les facteurs socio-culturels des manifestations d'intolérance xénophobe chez les adolescent.e.s et, d'autre part, sur le rôle de l'école dans la prévention de la pensée intolérante et la valorisation de la diversité ethno-culturelle. Membre de l'UMR URMIS, il est co-responsable du groupe de recherche " Jeunesse Éducation Altérité " et fellow de l'IC-Migrations de l'INED.

Maison des Sciences de l'Homme et de la Société Sud-Est, Pôle Universitaire Saint-Jean d'Angély 3, 24 avenue des diables bleus, F-06300 Nice

E-Mail: alessandro.bergamaschi@univ-cotedazur.fr

Catherine Blaya est professeure en sciences de l'éducation à l'Université Nice Côte d'Azur (UCA). Ses travaux de recherche portent sur le décrochage scolaire, le climat scolaire et la (cyber)violence entre jeunes. Ses derniers travaux s'intéressent plus particulièrement à la cyberhaine à caractère raciste, xénophobe ou religieux. Après avoir été Présidente de l'Observatoire International de la Violence à l'école de 2013 à 2018, elle a dirigé le Laboratoire Accrochages Scolaires et Alliances Éducatives à la Haute École Pédagogique du canton de Vaud. Elle est actuellement directrice de l'Institut National du Professorat et de l'Éducation.

Maison des Sciences de l'Homme et de la Société Sud-Est, Pôle Universitaire Saint-Jean d'Angély 3, 24 avenue des diables bleus, F-06300 Nice

E-Mail: catherine.blaya@univ-cotedazur.fr

Jimmy Stef est doctorant en sociologie, contractuel CNRS avec mobilité internationale à l'Université Côte d'Azur. Ses travaux de recherche portent sur l'éducation internationale et le phénomène de l'internationalisation de l'enseignement supérieur en Europe et en Asie du Sud-Est (Malaisie et Singapour). Ils sont également axés sur les stratégies institutionnelles et gouvernementales en matière de modèles éducatifs et sur la formation des élites internationales. Il est membre de deux laboratoires de recherche : à l'Unité de recherche Migrations et Sociétés (URMIS) et à l'Institut de recherche sur l'Asie du Sud-Est Contemporaine (IRASEC) à Bangkok (Thaïlande). Maison des Sciences de l'Homme et de la Société Sud-Est, Pôle Universitaire Saint-Jean d'Angély 3, 24 avenue des diables bleus, F-06300 Nice

E-Mail: jimmy.stef@univ-cotedazur.fr

Francesco Arcidiacono est professeur dans le domaine " Développement et interactions sociales " à la Haute École Pédagogique BEJUNE en Suisse. Au sein de la même institution, il dirige le Département de la recherche et le Centre de soutien et promotion de la recherche (CeSPRe). Ses travaux abordent les questions liées au développement au sein d'interactions sociales et à l'analyse de pratiques socio-culturelles en contexte d'éducation.

Haute Ecole Pédagogique - BEJUNE, Route de Moutier 14, CH-2800 Delémont

E-Mail: francesco.arcidiacono@hep-bejune.ch 